\title{
Distribuição de frequência da chuva para região Centro-Sul do Ceará, Brasil $^{1}$
}

\author{
Frequency distribution of rainfall for the South-Central region of Ceará, Brazil
}

Ítalo Nunes Silva ${ }^{2 *}$, Joaquim Branco de Oliveira $^{3}$, Larissa de Oliveira Fontes ${ }^{4}$ Francisco Dirceu Duarte Arraes ${ }^{5}$

\begin{abstract}
RESUMO - Foram analisadas sete distribuições de probabilidade Exponencial, Gama, Log-normal, Normal, Weibull, Gumbel e Beta para a chuva mensal e anual na região Centro-Sul do Ceará, Brasil. Para verificação dos ajustes dos dados às funções densidade de probabilidade foi utilizado o teste não-paramétrico de Kolmogorov-Smirnov com nível de 5\% de significância. Os dados de chuva foram obtidos da base de dados da SUDENE registrados durante o período de 1913 a 1989. Para a chuva total anual teve ajuste satisfatório dos dados às distribuições Gama, Gumbel, Normal e Weibull e não ocorreu ajuste às distribuições Exponencial, Log-normal e Beta. Recomenda-se o uso da distribuição Normal para estimar valores de chuva provável anual para a região, por ser um procedimento de fácil aplicação e também pelo bom desempenho nos testes. A distribuição de frequência Gumbel foi a que melhor representou os dados de chuva para o período mensal, com o maior número de ajustes no período chuvoso. No período seco os dados de chuva foram melhores representados pela distribuição Exponencial.
\end{abstract}

Palavras-chave: Chuvas. Climatologia agrícola. Precipitação (meteorologia) - previsão.

\begin{abstract}
Seven probability distributions were analysed: Exponential, Gamma, Log-Normal, Normal, Weibull, Gumbel and Beta, for monthly and annual rainfall in the south-central region of Ceará, Brazil. In order to verify the adjustments of the data to the probability density functions, the non-parametric Kolmogorov-Smirnov test was used with a 5\% level of significance. The rainfall data were obtained from the database at SUDENE, recorded from 1913 to 1989. For the total annual rainfall, adjustment of the data to the Gamma, Gumbel, Normal and Weibull distributions was satisfactory, and there was no adjustment to the Exponential, Log-normal and Beta distributions. Use of Normal distribution is recommended to estimate the values of probable annual rainfall in the region, this being a procedure of easy application, performing well in the tests. The Gumbel frequency distribution was the one that best represented rainfall data over a monthly period, with the highest number of adjustments during the rainy season. In the dry season, rainfall data were best represented by an exponential distribution.
\end{abstract}

Key words: Rains. Agricultural climatology. Precipitation (meteorology) - prediction.

\footnotetext{
* Autor para correspondência

'Recebido para publicação em 30/01/2012; aprovado em 07/02/2013

Parte da Monografia do primeiro autor, apresentada ao Instituto Federal de Educação, Ciência e Tecnologia do Ceará - Campus Iguatu ${ }^{2}$ Programa de Pós-Graduação em Fitotecnia da Universidade Federal Rural do Semi-Árido/UFERSA, Av. Francisco Mota, 572, Bairro Costa e Silva, Mossoró-RN, Brasil, 59.625-900, italonunessilva@gmail.com

${ }^{3}$ Programa de Pós-Graduação em Fitotecnia da Universidade Federal Rural do Semi-Árido/UFERSA, Prof. M. Sc. em Meteorologia Agrícola do Instituto Federal de Educação, Ciência e Tecnologia do Ceará - Campus Iguatu, Rodovia Iguatu, Várzea Alegre, Km 05, Iguatu-CE, Brasil, 63.500-000, joaquimbrancodeoliveira@gmail.com

${ }^{4}$ Programa de Pós-Graduação em Agronomia/Fitotecnia da Universidade Federal do Ceará/UFC, Fortaleza-CE, Brasil, larissafontesjp@ @otmail.com ${ }^{5}$ Programa de Pós-Graduação em Engenharia de Sistemas Agrícolas, Departamento de Engenharia de Biossistemas/ESALQ/USP, Piracicaba-SP, Brasil, dirceuarraes@gmail.com
} 


\section{INTRODUÇÃO}

A estimativa da chuva com determinado nível de probabilidade é de suma importância para o planejamento agrícola, possibilitando a previsão da melhor época de preparo do solo, colheita, semeadura, aplicação de adubos, defensivos, e lâmina suplementar de irrigação (ÁVILA; MELLO; VIOLA, 2009). A água é responsável por grande parte do desenvolvimento agrário de regiões onde os recursos hídricos são escassos, sendo a chuva a forma mais econômica e ambientalmente adequada de uso da água na agricultura (VIEIRA et al., 2010).

O conhecimento do comportamento e da distribuição das precipitações fornece subsídios para determinar períodos críticos predominantes numa determinada região e condições de fornecer informações que visem reduzir as consequências causadas pelas flutuações do regime pluviométrico, seja pelo emprego da irrigação ou pela implantação de culturas adaptadas à sazonalidade deste regime (JUNQUEIRA JUNIOR et al., 2007). De maneira geral, os projetos de irrigação são dimensionados visando suprir todas as necessidades hídricas da planta, sem levar em conta a parcela provável de precipitação. $\mathrm{O}$ dimensionamento de sistemas de irrigação baseia-se, geralmente, em previsões do uso de água pelas culturas. A utilização de estimativas da necessidade de água das culturas sem nenhum critério, ou resultante de modelos, nem sempre são capazes de proporcionar resultados confiáveis.

O aproveitamento dos recursos hídricos de forma adequada requer o conhecimento das probabilidades de ocorrência das chuvas, sendo isto possível com o uso das funções de distribuição de probabilidade de ocorrência (CATALUNHA et al., 2002). Ainda de acordo com Catalunha et al. (2002), o uso de funções densidade de probabilidade está diretamente ligado à natureza dos dados a que ela se relaciona. Algumas têm boa capacidade de estimação para pequeno número de dados, outras requerem grande série de observações. Devido ao número de parâmetros de sua equação, algumas podem assumir diferentes formas, enquadrando-se em um número maior de situações, ou seja, são mais flexíveis. Estudos de ajustes de função de distribuição de probabilidade ou estimativas de probabilidade usando funções de distribuição de probabilidade teóricas em relação a um conjunto de elementos climáticos têm sido desenvolvidos, enfatizando os benefícios no planejamento de atividades que minimizem riscos climáticos (ARRAES et al., 2009; RIBEIRO et al., 2007; SILVA et al., 1998; VIANA et al., 2005).

O objetivo deste trabalho é testar o ajuste dos dados de chuva mensal e anual da região Centro-Sul do estado do Ceará a diferentes funções de distribuição de probabilidade.

\section{MATERIAL E MÉTODOS}

A área de estudo envolve a região Centro-Sul do Ceará que ocupa uma área de $142.016 \mathrm{~km}^{2}$ e corresponde a 9,6\% da Região Nordeste e $2 \%$ do país (ANDRADE; SILVEIRA; AZEVEDO et al., 2003). Foram utilizados dados de chuva de 1913 a 1989 coletados em postos pluviométricos localizados nas cidades de Acopiara, Cariús, Cedro, Icó, Iguatu, Lima Campos, Jaguaribe, Orós, Solonópole e Várzea Alegre (Tabela 1), obtidos junto à Superintendência do Desenvolvimento do Nordeste (SUDENE).

Os postos selecionados para o estudo situam-se na região fisiográfica do sertão nordestino cuja classificação climática de Köppen é do tipo BSw'h', clima quente e semi-árido, caracterizado pela insuficiência das chuvas, com temperaturas elevadas acarretando numa forte evaporação, e tendo apenas duas estações climáticas

Tabela 1 - Localização e características dos postos em estudo para região Centro Sul do Ceará

\begin{tabular}{rcccccc}
\hline $\mathrm{N}^{\circ}$ & Posto & $\mathrm{N}^{\circ}$ Posto & Latitude & Longitude & Altitude (m) & Período (anos) \\
\hline 1 & Acopiara & 3820369 & $6^{\circ} 11^{\prime} \mathrm{S}$ & $39^{\circ} 40^{\prime} \mathrm{W}$ & 270 & $1913-1989$ \\
2 & Cariús & 3831006 & $6^{\circ} 32^{\prime} \mathrm{S}$ & $39^{\circ} 29^{\prime} \mathrm{W}$ & 230 & $1913-1989$ \\
3 & Cedro & 3831287 & $6^{\circ} 36^{\prime} \mathrm{S}$ & $39^{\circ} 04^{\prime} \mathrm{W}$ & 246 & $1913-1989$ \\
4 & Icó & 3822832 & $6^{\circ} 25^{\prime} \mathrm{S}$ & $38^{\circ} 51^{\prime} \mathrm{W}$ & 160 & $1913-1989$ \\
5 & Iguatu & 3821742 & $6^{\circ} 22^{\prime} \mathrm{S}$ & $39^{\circ} 18^{\prime} \mathrm{W}$ & 213 & $1913-1989$ \\
6 & Jaguaribe & 3812779 & $5^{\circ} 53^{\prime} \mathrm{S}$ & $38^{\circ} 37 \mathrm{~W}$ & 120 & $1913-1989$ \\
7 & Lima Campos & 3822808 & $6^{\circ} 25^{\prime} \mathrm{S}$ & $38^{\circ} 58^{\prime} \mathrm{W}$ & 180 & $1913-1989$ \\
8 & Orós & 3822518 & $6^{\circ} 16^{\prime} \mathrm{S}$ & $38^{\circ} 55^{\prime} \mathrm{W}$ & 188 & $1913-1989$ \\
9 & Solonópole & 3811498 & $5^{\circ} 44^{\prime} \mathrm{S}$ & $39^{\circ} 01^{\prime} \mathrm{W}$ & 170 & $1913-1989$ \\
10 & Várzea Alegre & 3831543 & $6^{\circ} 47^{\prime} \mathrm{S}$ & $39^{\circ} 18^{\prime} \mathrm{W}$ & 345 & $1913-1989$ \\
\hline
\end{tabular}


bem definidas durante o ano: uma chuvosa e outra seca (ARAÚJO et al., 2010; ARRAES et al., 2009).

A homogeneidade dos dados de chuva foi verificada pelo teste de "Run" conforme procedimento descrito por Thom (1966). Foram analisados os totais anuais e mensais de chuva.

\section{As distribuições utilizadas na análise foram:}

a) Distribuição exponencial: geralmente aplicada a dados com forte assimetria, ou seja, apresentando uma forma de "J" invertido. Sua função densidade de probabilidade é assim descrita (CATALUNHA et al., 2002):

$$
\mathrm{F}(\mathrm{x})=\frac{\exp \left(-\frac{\mathrm{x}}{\overline{\mathrm{x}}}\right)}{\overline{\mathrm{x}}}
$$

Sua função de distribuição de probabilidade é do tipo:

$$
F(x)=\int_{0}^{x}\left(\frac{\exp \left(-\frac{x}{\bar{x}}\right)}{\bar{x}}\right)=1-\exp \left(-\frac{x}{\bar{x}}\right)
$$

O único parâmetro da distribuição como visto, é a média. Com isso, a função cumulativa de probabilidade assume a forma de:

$$
\mathrm{F}(\mathrm{x})=1-\exp \left(-\frac{\mathrm{x}}{\overline{\mathrm{x}}}\right)
$$

A distribuição exponencial é um caso particular da distribuição Gama com o parâmetro $\alpha=1$.

b) Distribuição Beta: é uma distribuição definida no intervalo $(0,1)$ com a seguinte função densidade de probabilidade (ASSIS; ARRUDA; PEREIRA, 1996):

$$
F(x)=\frac{x^{(\alpha-1)(1-x)^{\beta-1}}}{\beta^{(\alpha, \beta)}}
$$

em que, $0<\mathrm{x}<1$ e $\alpha, \beta>0$.

A função beta $B(\alpha, \beta)$ é definida por:

$$
B(\alpha, \beta)=\int_{0}^{1} x^{\alpha-1}(1-x)^{\beta-1} d x
$$

c) Distribuição Gama: se $\mathrm{x}$ for uma variável aleatória contínua, tal que $(0<\mathrm{x}<\infty)$, com distribuição Gama de parâmetros $\alpha>0$ e $\beta>0$, então a sua função densidade de probabilidade é definida como (JUNQUEIRA JÚNIOR et al., 2007):

$$
\mathrm{F}(\mathrm{x})=\frac{1}{\beta^{\alpha} \Gamma(\alpha)} \mathrm{x}^{\alpha-1} \exp \left(\frac{\mathrm{x}}{\beta}\right) \quad ; \text { para } 0<\mathrm{x}<\infty
$$

Algumas formas de estimar os parâmetros da distribuição Gama foram desenvolvidas, contribuindo, junto com a sua flexibilidade de formas, para sua utilização em diversas áreas (HAAN, 1977; CATALUNHA et al., 2002). Os parâmetros $\alpha$ e $\beta$ foram calculados pelo método da máxima verossimilhança, como propostas por Thom (1958):

$\hat{\alpha}=\frac{1+\sqrt{1+\frac{4\left[\ln (\mathrm{x})-\mathrm{x}_{\mathrm{g}}\right]}{3}}}{4\left[\ln (\overline{\mathrm{x}})-\mathrm{x}_{\mathrm{g}}\right]}$

O estimador do parâmetro $\beta$ poderá ser obtido por:

$$
\hat{\beta}=\frac{\bar{x}}{\hat{\alpha}}
$$

em que, $\mathrm{x}$ é a média aritmética; $\mathrm{x}_{\mathrm{g}}$ é a média geométrica das observações.

Sendo $F(x)$ a probabilidade de ocorrência de um evento menor ou igual a $x$, pode-se escrever que a função de distribuição acumulada de probabilidade é representada pela função Gama incompleta, segundo Thom (1958):

$$
F(x)=\frac{1}{\beta^{\alpha} \Gamma(\alpha)} \int_{0}^{x} u^{\alpha-1} \exp \left(-\frac{u}{\beta}\right) d u
$$

em que, $\mathrm{F}(\mathrm{x})$ é a probabilidade de ocorrer um valor menor ou igual a $\mathrm{x}$; $\mathrm{x}$ é a variável aleatória contínua; $\Gamma(\alpha)$ é a função Gama do parâmetro alfa; $\alpha$ é o parâmetro de forma da variável aleatória $x ; \beta$ é o parâmetro de escala da variável aleatória $\mathrm{x}$; $\mathrm{u}$ é a variável aparente utilizada para integração.

A função de distribuição acumulada da distribuição Gama possui uma integral, que pode ser resolvida por métodos numéricos ou pelo desenvolvimento de uma expansão em série (Equação 10 e 11) conforme Assis, Arruda e Pereira (1996):

$$
\begin{aligned}
& F(t)=\frac{1}{\Gamma(\alpha)} \int_{0}^{t} t^{\alpha-1} \exp (-t) d t \\
& F(t)=\frac{t^{\alpha}}{\alpha \Gamma(\alpha) \exp (t)}\left[1+\frac{t}{\alpha+1}+\frac{t^{2}}{(\alpha+1)(\alpha+2)}+\frac{t^{3}}{(\alpha+1)(\alpha+2)(\alpha+3)}+\ldots\right]
\end{aligned}
$$


em que, $\mathrm{t}$ - $\mathrm{x} / \beta$, é uma aproximação da distribuição Gama por expansão em série.

d) Distribuição Log-normal: conforme Ribeiro et al. (2007), a função densidade da distribuição Log-normal a dois e três parâmetros (Equação 12).

$$
F(x)=\frac{1}{(x-a) \sigma \sqrt{2 \pi}} \exp \left(-\frac{[\ln (x-a)-\mu]^{2}}{2 \sigma^{2}}\right)
$$

em que, $F(x)$ é a função densidade de probabilidade da variável; $\mu$ é a média dos logaritmos da variável $\mathrm{x}$; $\sigma$ é o desvio-padrão dos logaritmos da variável x; a é o limite inferior da amostra.

Para encontrar a probabilidade de que uma variável aleatória $\mathrm{x}$ tendo distribuição Log-normal, assuma valores entre a e $b(a \leq x \leq b)$, tem-se:

$$
F(a \leq x \leq b)=\int_{a}^{b} \frac{1}{x \sigma \sqrt{2 \pi}} \exp \left(-\frac{[\ln (x-a)-\mu]^{2}}{2 \sigma^{2}}\right) d x
$$

O valor de "a" pode ser zero, quando se considera a distribuição Log-normal a dois parâmetros, ou um valor mínimo da série, quando se considera Lognormal a três parâmetros. O valor de "b" pode ser o da variável aleatória, quando se considera a probabilidade cumulativa de ocorrência daquele valor (CATALUNHA et al., 2002).

e) Distribuição Normal: tem função densidade de probabilidade da seguinte forma (ASTOLPHO et al., 2005; MARTIN; STORCK; DOURADO NETO, 2007):

$$
\mathrm{F}(\mathrm{x})=\frac{1}{\sigma \sqrt{2 \pi}} \exp \left(-\frac{(\mathrm{x}-\mu)^{2}}{2 \sigma^{2}}\right) ; \text { para }-\infty<\mathrm{x}<+\infty(14)
$$

em que, $\mu$ é a média.

A probabilidade de que $\mathrm{x}$ assuma valores menores ou iguais a um dado $\mathrm{x}$ quando $\mathrm{x}$ é $\mathrm{N}\left(\mu, \sigma^{2}\right)$, é estimada por:

$$
F(x)=\frac{1}{\sigma \sqrt{2 \pi}} \int_{-x}^{x} \exp \left(\frac{(x-\mu)^{2}}{2 \sigma^{2}}\right) d x
$$

Essa equação não pode ser resolvida analiticamente sem o uso de métodos de integração aproximada. Por esse motivo, usa-se a transformação $\mathrm{Z}=(\mathrm{x}-\mu) / \sigma$, a variável $\mathrm{Z}$ tem distribuição Normal com média zero e variância um $[\mathrm{N}(0,1)]$. A variável $\mathrm{Z}$ é chamada variável reduzida e a Equação 15 pode ser reescrita na seguinte forma:

$$
F(Z)=\frac{1}{\sqrt{2 \pi}} \int_{-\infty}^{Z} \exp \left(-\frac{Z^{2}}{2}\right) d z ; \text { para }-\infty \leq Z \leq+\infty
$$

f) Distribuição Weibull: conforme Catalunha et al. (2002) sua função de densidade de probabilidade mais comumente apresentada conforme a Equação 17:

$$
\mathrm{F}(\mathrm{x})=\frac{\gamma}{\beta}\left(\frac{\mathrm{x}-\alpha}{\beta}\right)^{\gamma-1} \exp \left[-\left(\frac{\mathrm{x}-\alpha}{\beta}\right)^{\gamma}\right] ; \text { para } \mathrm{x} \geq \alpha
$$

sendo $\mathrm{F}(\mathrm{x})=0$ para outros intervalos, e $\alpha \geq 0, \beta>0, \gamma>0$ são os parâmetros da distribuição.

g) Distribuição Gumbel: ou distribuição tipo I de Fisher-Tippet tem sua função de densidade de probabilidade conforme Beijo, Muniz e Castro Neto (2005) e Naghettini; Pinto, (2007) da seguinte forma:

$$
\mathrm{F}(\mathrm{x})=\frac{1}{\beta} \exp \left(-\frac{\mathrm{x}-\alpha}{\beta}\right) \exp \left(-\exp \left(-\frac{x-\alpha}{\beta}\right)\right.
$$

em que, $\alpha$ é o parâmetro de posição; $\beta$ é o parâmetro de escala.

Sua função de distribuição acumulada é dada pela Equação 19, sendo que o sinal \pm no segundo expoente, refere-se aos valores extremos máximos (sinal negativo) e mínimos (sinal positivo).

$\mathrm{F}(\mathrm{x})=\exp \left(-\exp \pm\left(\frac{\mathrm{x}-\alpha}{\beta}\right)\right.$

A aderência das distribuições aos dados observados foi verificada pelo teste de KolmogorovSmirnov (KS) conforme procedimentos descritos por Assis, Arruda e Pereira (1996). Este teste foi introduzido por Kolmogorov e Smirnov como metodologia para sua aplicação, pode-se considerar $\mathrm{F}(\mathrm{x})$ a proporção dos valores esperados menores ou iguais a $\mathrm{x}$ e $\mathrm{S}(\mathrm{x})$ é a proporção dos valores observados menores ou iguais a $\mathrm{x}$, portando utilizasse o módulo do desvio máximo observado (Dobs):

Dobs = Máx $[\mathrm{F}(\mathrm{x})-\mathrm{S}(\mathrm{x})]$

Para isto compara-se o valor do desvio máximo observado com o desvio máximo critico (Dcrit); se Dobs for menor que Dcrit, existe concordância entre as frequências observadas e esperadas, a amostra provêm de uma população que segue a distribuição de probabilidade testada. 


\section{RESULTADOS E DISCUSSÃO}

Os valores de chuva mensal da região em estudo apresentaram dois períodos distintos, sendo o primeiro correspondente aos meses chuvosos (janeiro a maio) e o segundo correspondente aos meses secos (junho a dezembro). Períodos esses coincidentes com as características sazonais da região estudada.

No período chuvoso os melhores ajustes dos dados foram para as distribuições Gumbel, em 90\% dos postos, no mês abril, e 50\% dos postos em janeiro e Weibull, em $60 \%$ dos postos para fevereiro e $50 \%$ dos postos para maio (Tabela 2), sendo as únicas distribuições que tiveram aderências em todos os meses desse período. Resultado semelhante ocorreu para a cidade de Santa Maria (RS), onde Silva et al. (2007) perceberam o bom comportamento dos dados à distribuição Weibull nas épocas chuvosas. Já para Táchira, na Venezuela, as distribuições de Gumbel e Weibull não apresentaram resultados satisfatórios (LYRA et al., 2006).

No período seco, predominou a distribuição exponencial com o ajuste dos dados em todos os meses que correspondem ao período seco chegando a $80 \%$ dos ajustes nos meses de junho e novembro, seguida pela distribuição beta com $30 \%$ de ajustes no mês de outubro sem nenhum ajuste em junho (Tabela 2). O bom desempenho da distribuição exponencial pode ser explicado pela maior frequência observada nas classes iniciais, decrescendo suavemente, em forma de " $\mathrm{J}$ " invertido, com forte assimetria (LYRA et al., 2006). Blain et al. (2007) encontraram resultados semelhantes nos meses secos estudando a chuva mensal no posto meteorológico do Instituto Agronômico, em Campinas (SP).
Para os totais anuais de chuva destaca-se a distribuição Normal (Tabela 2) com 50\% dos ajustes seguida pelas distribuições Gama, Weibull e Gumbel com 20; 20 e 10\% das aderências respectivamente. Em estudo realizado para a cidade de Tangará da Serra (MT), Martins et al. (2010), utilizando a distribuição de probabilidade Gama, encontraram boa adequação dos dados à distribuição de probabilidade testada segundo teste KS, resultados semelhantes foram encontrados por Soccol et al. (2009) em estudo realizado para a cidade de Lages, Santa Catarina, Murta et al. (2005) em estudo realizado para o sudoeste da Bahia e por Beijo et al. (2003) com a distribuição de Weibull, em períodos mensais para a cidade de Jaboticabal (SP).

Em julho foi observado o bom desempenho da exponencial seguida pela de Gama nos postos de Icó, Jaguaribe, Lima Campos e Várzea Alegre (Tabela 3). Isso se deve em parte à distribuição exponencial ser um caso particular da gama, com seu parâmetro de forma ( $\alpha$ ) igual a 1 (CATALUNHA et al., 2002). Estudando o comportamento dos períodos secos e chuvosos no município de Garanhuns (PE), Andrade et al. (2008) concluíram que os dados apresentaram bom ajuste à distribuição Gama, corroborando com Ribeiro et al. (2007) que encontram também resultados satisfatórios para estimar a precipitação provável para a região de Barbacena (MG).

Destaca-se o bom desempenho da distribuição Gumbel no mês de abril (Tabela 3), período chuvoso, onde só não houve ajuste dos dados do posto Jaguaribe. Mesmos resultados foram encontrados por Beijo, Muniz e Castro Neto (2005) estudando a distribuição Gumbel para a região de Lavras (MG), onde os melhores resultados foram nos meses com maior índice de chuva.

Tabela 2 - Porcentagem de aderências das funções de distribuição de probabilidade de chuva mensal e anual nos postos de Acopiara, Cariús, Cedro, Icó, Iguatu, Jaguaribe, Lima Campos, Orós, Solonópole e Várzea Alegre, pelo teste de Kolmogorov-Smirnov (KS) com nível de 5\%

\begin{tabular}{|c|c|c|c|c|c|c|c|c|c|c|c|c|c|}
\hline \multirow{2}{*}{ FDP } & \multicolumn{13}{|c|}{ Porcentagem de aderência } \\
\hline & Jan. & Fev. & Mar. & Abr. & Mai. & Jun. & Jul. & Ago. & Set. & Out. & Nov. & Dez. & AN \\
\hline EX & 20 & 0 & 0 & 0 & 0 & 80 & 40 & 60 & 60 & 50 & 80 & 70 & 0 \\
\hline GA & 10 & 10 & 0 & 0 & 10 & 0 & 40 & 10 & 0 & 0 & 0 & 0 & 20 \\
\hline GU & 50 & 20 & 20 & 90 & 40 & 0 & 0 & 0 & 10 & 10 & 0 & 0 & 10 \\
\hline NO & 0 & 0 & 40 & 0 & 0 & 0 & 0 & 0 & 10 & 0 & 0 & 0 & 50 \\
\hline $\mathrm{LN}$ & 0 & 10 & 0 & 0 & 0 & 0 & 0 & 10 & 0 & 0 & 0 & 0 & 0 \\
\hline WE & 20 & 60 & 40 & 10 & 50 & 20 & 0 & 0 & 0 & 0 & 0 & 10 & 20 \\
\hline $\mathrm{BE}$ & 0 & 0 & 0 & 0 & 0 & 0 & 20 & 10 & 10 & 30 & 20 & 20 & 0 \\
\hline SIG & 0 & 0 & 0 & 0 & 0 & 0 & 0 & 10 & 10 & 10 & 0 & 0 & 0 \\
\hline
\end{tabular}

FDP - Funções de distribuição de probabilidade; AN - Anual; EX - Exponencial; GA - Gama; GU - Gumbel; NO - Normal; LN - Log-normal; WE Weibull; BE - Beta; SIG - Significativo a 5\%: os dados amostrais não se ajustaram a nenhuma das distribuições avaliadas 
Tabela 3 - Funções de distribuições de probabilidade que melhor se ajustaram a chuva mensal e anual nos postos de Acopiara, Cariús, Cedro, Icó, Iguatu, Jaguaribe, Lima Campos, Orós, Solonópole e Várzea Alegre, pelo teste de Kolmogorov-Smirnov (KS) com nível de 5\%

\begin{tabular}{lllllllllllllll}
\hline \multirow{2}{*}{ EST } & \multicolumn{1}{c}{} & \multicolumn{1}{c}{ Jan. } & Fev. & Mar. & Abr. & Mai. & Jun. & Jul. & Ago. & Set. & Out. & Nov. & Dez. & AN \\
\hline ACO & GU & WE & GU & GU & WE & EX & BE & LN & EX & BE & BE & EX & NO \\
CAR & WE & WE & WE & GU & WE & WE & EX & EX & BE & EX & EX & EX & GU \\
CED & WE & WE & WE & GU & WE & EX & EX & EX & GU & EX & EX & EX & GA \\
ICO & GU & WE & WE & GU & WE & EX & GA & GA & EX & BE & EX & EX & GA \\
IGT & EX & GU & NO & GU & GU & EX & EX & EX & EX & BE & EX & BE & NO \\
JAG & GU & GA & NO & WE & WE & WE & GA & EX & $*$ & EX & BE & WE & WE \\
LMC & GU & LN & NO & GU & GU & EX & GA & EX & EX & EX & EX & EX & WE \\
ORO & GU & GU & GU & GU & GA & EX & BE & BE & EX & GU & EX & EX & NO \\
SOL & EX & WE & NO & GU & GU & EX & EX & EX & NO & $*$ & EX & BE & NO \\
VZA & GA & WE & WE & GU & GU & EX & GA & $*$ & EX & EX & EX & EX & NO \\
\hline
\end{tabular}

EST - Estação; AN - Anual; ACO - Acopiara; CAR - Cariús; CED - Cedro; ICO - Icó; IGT - Iguatu; JAG - Jaguaribe; LMC - lima campos; ORO - Orós; SOL - Solonópole; VZA - Várzea alegre; EX - Exponencial; GA - Gama; GU - Gumbel; NO - Normal; LN - Log-normal; WE - Weibull; BE - Beta; *Significativo a 5\%: os dados amostrais não se ajustaram a nenhuma das distribuições avaliadas

Em março, a distribuição Normal apresentou bom desempenho nos postos Iguatu, Jaguaribe, Lima Campos e Solonópole (Tabela 3), provavelmente por ser o mês o qual raramente se verifica ausência de chuva. Junqueira Júnior et al. (2007), estimando precipitação provável com a distribuição Normal, verificaram resultados não satisfatórios em seu estudo para a região de Madre Deus (MG).

A distribuição Log-normal (Tabela 3) obteve maior índice de rejeição, tanto para os meses chuvosos como para os mais secos. Observou-se ajuste dos dados de chuva à distribuição apenas nos postos de Acopiara e Lima Campos em agosto e fevereiro respectivamente. Junqueira Júnior et al. (2007) em estudo realizado para a região de Madre de Deus encontraram a distribuição Lognormal como a mais adequada para períodos mensais.

Para os meses de agosto, setembro e outubro, nos postos de Jaguaribe, Solonópole e Várzea Alegre não houve ajuste dos dados a nenhuma das distribuições testadas. Lyra et al. (2006) também não observou ajuste dos dados a nenhuma distribuição testada para os mesmos meses em Táchira, Venezuela.

\section{CONCLUSÕES}

1. A chuva total anual teve ajuste satisfatório para as distribuições Gama, Gumbel, Normal e Weibull e não se ajustou às distribuições Exponencial, Lognormal e Beta;
2. Recomenda-se o uso da distribuição Normal para estimar valores de chuva provável anual para a região, por ser um procedimento de fácil aplicação e também pelo bom desempenho nos testes;

3. A distribuição de frequência Gumbel foi a que melhor representou os dados de chuva para o período mensal, com o maior número de ajustes no período chuvoso;

4. No período seco os dados de chuva foram melhores representados pela distribuição Exponencial.

\section{REFERÊNCIAS}

ANDRADE, A. R. et al. Estudo do comportamento de períodos secos e chuvosos no município Garanhuns, PE, para fins de planejamento agrícola. Pesquisa Aplicada e Agrotecnologia, v. 1, n. 1, p. 55-61, 2008.

ANDRADE, E. M.; SILVEIRA, S. S.; AZEVEDO, B. M. Investigação da estrutura multivariada da evapotranspiração na região Centro-Sul do Ceará pela análise de componentes principais. Revista Brasileira de Recursos Hídricos, v. 8, n. 1, p. 39-44, 2003.

ARRAES, F. D. D. et al. Estimativa do balanço hídrico para as condições climáticas Iguatu, Ceará, usando modelo estocástico. Revista Brasileira de Agricultura Irrigada, v. 3, n. 2, p. 78-87, 2009.

ARAÚJO, E. M. et al. Aplicação de seis distribuições de probabilidade a séries de temperatura máxima em Iguatu - CE. Revista Ciência Agronômica, v. 41, n. 1, 36-45, 2010. 
ASSIS, F. N.; ARRUDA, H. V.; PEREIRA, A. R. Aplicações de estatística à climatologia: teoria e prática. Pelotas: UFPel, 1996. $161 \mathrm{p}$.

ASTOLPHO, F. et al. Regionalização de riscos de ocorrência de temperaturas mínimas absolutas anuais para o estado de São Paulo com base em modelos probabilísticos e digitais de elevação. Bragantia, v. 64, n. 1, p. 139-148, 2005.

ÁVILA, L. F.; MELLO, C. R.; VIOLA, M. R. Mapeamento da precipitação mínima provável para o sul de Minas Gerais. Revista Brasileira de Engenharia Agrícola e Ambiental, v. 13, p. 906-915, 2009. Suplemento.

BEIJO, L. A. et al. Estudo da precipitação máxima em Jaboticabal (SP) pela distribuição de Gumbel utilizando dois métodos de estimação dos parâmetros. Revista Brasileira de Agrometeorologia, v. 11, n. 01, p. 141-147, 2003.

BEIJO, L. A.; MUNIZ, J. A.; CASTRO NETO, P. Tempo de retorno das precipitações máximas em lavras (MG) pela distribuição de valores extremos do tipo I. Ciência e Agrotecnologia, v. 29, n. 3, p.657-667, 2005.

BLAIN, G. C. et al. Distribuição temporal da precipitação pluvial mensal observada no posto meteorológico do instituto agronômico, em Campinas, SP. Bragantia, v. 66, n. 2, p. 347-355, 2007.

CATAlunHA, M. J. et al. Aplicação de cinco funções densidade de probabilidade a séries de precipitação pluvial no estado de Minas Gerais. Revista Brasileira de Agrometeorologia, v. 10, n. 1, p. 153-162, 2002.

JUNQUEIRA JÚNIOR, J. A. et al. Precipitação provável para a região de Madre de Deus, Alto Rio Grande: Modelos de probabilidades e valores característicos. Ciência e Agrotecnologia, v. 31, n. 3, p.842-850, 2007.

LYRA, G. B. I. I. et al. Regiões homogêneas e funções de distribuição de probabilidade da precipitação pluvial no estado de Táchira, Venezuela. Pesquisa Agropecuária Brasileira, v. 41, n. 2, p. 205-215, 2006.

MARTIN, T. N.; STORCK, L.; DOURADO NETO, D. Simulação estocástica da radiação fotossinteticamente ativa e da temperatura do ar por diferentes métodos. Pesquisa Agropecuária Brasileira, v. 42, n. 9, p. 1211-1219, 2007.
MARTINS, J. A. et al. Probabilidade de precipitação para a microrregião de Tangará da Serra, Estado do Mato Grosso. Pesquisa Agropecuária Tropical, v. 40, n. 3, p. 291-296, 2010.

MURTA, R. M. I. et al. Precipitação pluvial mensal em níveis de probabilidade pela distribuição gama para duas localidades do sudoeste da Bahia. Ciência e Agrotecnologia, v. 29, n. 5, p. 988-994, 2005.

NAGHETTINI, M.; PINTO, E. J. A. Hidrologia estatística. Belo Horizonte: CPRM, 2007. 552 p.

RIBEIRO, B. T. et al. Comparação de distribuições de probabilidade e estimativa da precipitação provável para região de Barbacena, MG. Ciência e Agrotecnologia, v. 31, n. 5, p. 1297-1302, 2007.

SILVA, F. C. et al. Distribuição e frequência da evapotranspiração de referência de Cruz das Almas, BA. Revista Brasileira de Engenharia Agrícola e Ambiental, v. 2, n. 3, p.284-286, 1998.

SILVA, J. C. et al. Análise de distribuição de chuva para Santa Maria, RS. Revista Brasileira de Engenharia Agrícola e Ambiental, v. 11, n. 1, p. 67-72, 2007.

SOCCOL, O. J.; CARDOSO, C. O.; MIQUELLUTI, D. J. Análise da precipitação mensal provável para o município de Lages, SC. Revista Brasileira de Engenharia Agrícola e Ambiental, v. 14, n. 6, p. 569-574, 2009.

THOM, H. C. S. A note on the gamma distribution. Monthly Weather Review, v. 86, p. 117-122, 1958.

THOM, H. C. S. Some methods of climatological analysis. Roma: FAO, 1966. 50 p.

VIEIRA, J. P. G. et al. Estudo da precipitação mensal durante a estação chuvosa em Diamentina, Minas Gerais. Revista Brasileira de Engenharia Agrícola e Ambiental, v. 14, n. 7, p. 762-767, 2010.

VIANA, T. V. A. et al. Análise estocástica dos déficits e dos excedentes hídricos mensais em Fortaleza, CE. Revista Ciência Agronômica, v. 36, n. 3, p. 391-395, 2005. 